\title{
Size Effects In the Crushing of Honeycomb Structures
}

\author{
Erik C. Mellquist* and Anthony M. Waas ${ }^{\dagger}$ \\ Composite Structures Laboratory, Aerospace Engineering Department, The University of Michigan, Ann Arbor, MI,
} 48109

\begin{abstract}
The effect of cell size on the out of plane compressive crushing response of circular cell polycarbonate honeycomb is presented. The results are a continuation of a study originally done by Mellquist and Waas, 2002. Experiments were conducted on hexagonal cell honeycombs ranging in size from 1 to 14 cells. The results show that no scaling factor can be determined for the crushing load based on sample size, but that stability appears to be a driving property. Specimen cross-section shapes that have an arrangement of cells with higher orders of symmetry provide greater stability and are less imperfection sensitive and therefore have higher crushing loads. Finite element analysis (FEA) was used to better understand the behavior of the honeycombs during crushing and to validate experimental results. An FEA model developed during the previous work (a 7 cell model) has been expanded to multiple sizes. The results are presented and show that the model can accurately predict how different size honeycombs will perform under crushing loads.
\end{abstract}

$\begin{array}{ll}E & =\text { Young's modulus } \\ N & =\text { number of circular cells in a honeycomb sample } \\ \square & =\text { Poisson's ratio } \\ \square_{\text {max }} & =\text { the maximum crushing stress of a honeycomb sample } \\ \square_{\text {plateau }} & =\text { the plateau crushing stress of a honeycomb sample }\end{array}$

\section{Introduction}

Sandwich structures (that employ a honeycomb core between two relatively thin skins) are desirable in several Nengineering applications that require high strength to weight ratios. Because of their ability to absorb large amounts of energy, they are also often used as a "cushion" against external loads. Honeycomb sandwich structures are currently being used in many engineering applications, both within and outside of aerospace engineering. Most studies in the literature are related to one of the attributes (high strength/weight or increased energy absorption) mentioned above. In the latter case, when detailed calculations warrant finding stresses and strains at the cell level, a numerical method, such as the finite element method is employed. In those instances, a large number of elements are required to perform the discrete cell level calculations. It is therefore desirable to develop a continuum level characterization for the honeycomb, as has been detailed in Ref. 1-3.

With regard to the development of a continuum model, one issue that has been overlooked is the scaling of honeycomb properties with respect to cell size. That is, one requires identifying certain geometrically based repeating units of the honeycomb (representative unit cell - RUC) and establishing the independence (or lack thereof) of honeycomb properties with respect to the number of such RUC's. By definition, RUC properties should be scalable for a large number of cells. This was the focus of work reported in Ref. 1, where preliminary results that focused on the size effects in the out of plane compressive crushing of circular cell polycarbonate (PC) honeycomb were presented. The current paper expands upon this study. The results from a new experimental program with several improvements over the original will be presented and discussed. The out of plane response as a function of

\footnotetext{
* Graduate Research Assistant, Aerospace Engineering, University of Michigan, Ann Arbor, MI 48109. Student Member, AIAA.

${ }^{\dagger}$ Professor of Aerospace Engineering, University of Michigan, Ann Arbor, MI 48109. Associate Fellow, AIAA.
} 
specimen size and shape will be discussed. In addition, a numerical model based on the finite element method and aimed at predicting sample behavior will be presented.

\section{Laboratory Tests}

\section{A. Honeycomb Test Samples}

Test samples were made of polycarbonate circular cell honeycomb purchased from Plascore, in Zeeland, MI. This is the same material that was studied in Ref. 1-3. The individual cells have a radius of 0.0625 inches and a wall thickness of $5.068 \times 10^{-3}$ inches. The honeycomb sample has an out of plane height of 1.0 inch. The polycarbonate, assumed isotropic, has the following material properties: Young's Modulus, E= $351.5 \mathrm{kSI}$, and Poisson's ratio $\square=0.3$. Test samples were carefully prepared individually by hand so that the external walls were free from broken cells and remnants of the cells they were cut away from. Samples were cut into twenty-four different sizes. The size was quantified by the number of cells $(\mathrm{N})$ that the sample contained. All sizes between one cell and fourteen cells $(\mathrm{N}=1-14)$ were studied. The honeycombs were attached directly to the loading frame by setting the ends of the honeycombs in $1 / 8^{\text {th }}$ inch of epoxy. The samples were placed under a light load while the epoxy was setting to insure that a strong bond was formed.

In the initial work ${ }^{1}$, which studied honeycombs ranging in size from 1 cell to 169 cells, pieces of flat aluminum were glued to the top and bottom of each honeycomb surface with epoxy in order to clamp them during the testing. These samples were placed within the loading frame, but were not attached to it.

The new setup guarantees that the honeycombs remained rigidly attached to the loading frame during testing. This allows for data to be recorded while the honeycombs are being unloaded. A comparison of the original and updated setup is shown in Figure 1.

\section{B. Test Method}

A screw driven testing machine was used to perform the crush experiments. Uniaxial out of plane compressive quasi-static loads were measured under displacement control conditions with the use of a load cell. The rigid load frame crush platen movement and a LVDT were used to calculate the macroscopic strain ${ }^{\square}$ based on the total contraction at any point in time during crushing. Measurements were taken at a sampling rate greater than $2 \mathrm{~Hz}$.

Each sample was loaded until it was crushed at least $1 / 4$ of an inch (or a macroscopic strain of 0.33 ). Figure 2 shows pictures of undeformed and deformed honeycombs containing seven cells $(\mathrm{N}=7)$. After the honeycombs were loaded they were immediately unloaded until the load decreased to zero. In several tests the honeycombs were loaded and unloaded multiple times.

\section{Material Behavior}

The behavior of all the samples was characterized by an initial period of stiff linear response followed by the achievement of a maximum stress $\left(\square_{\max }\right)$. During this period, the load rose very quickly until a macroscopic strain of about 0.02 was reached. At this point the load reached its maximum value. When this occurred, there was no noticeable deformation. Immediately after the maximum load was reached, a well-defined localized buckle formed around the circumference of the honeycombs. The buckle pattern was located a short distance (approximately 0.2 inches from the top edge of the specimens, in the crush direction). At this point, the load dropped very quickly. After this, the single buckle zone began expanding along the crushing axis of the honeycombs, and a sizable region of crush (a crush zone) at a constant state of stress (plateau stress $\left(\square_{\text {plateau }}\right)$ ) was observed. After a plateau stress had been reached the honeycombs were unloaded until the load reached zero. During the unloading period, the crush zones lengthened slightly. After loading and unloading were complete the honeycombs remained significantly shorter than they were originally, indicating that energy had been absorbed. A typical response diagram in macroscopic stress-macroscopic strain space is as indicated in Figure 3 ( $\square$ max and $\square_{\text {plateau }}$ are also shown) for a honeycomb sample with $\mathrm{N}=7$.

\section{Experimental Results}

\section{A. Scaling Results}

\footnotetext{
Macroscopic strain is defined as crush displacement divided by the original length of the specimen. The resulting macroscopic stress-strain curve is similar to the $\mathrm{P}_{-}$curve in a stability problem.
} 
A total of approximately 150 specimens were tested (from both the first and second study). The results were analyzed by examining the macroscopic stress-stain response of each honeycomb sample tested. In order to calculate stress, the loading area of a honeycomb sample was considered to be the area contained within the outer edges of the sample.

We focused our study on two major properties of every test, $\square_{\max }$ and $\square_{\text {plateau. It }}$. was previously reported ${ }^{1}$ that $\square_{\max }$ was highest for $\mathrm{N}=7$. The results of this set of tests (with the load introduction boundaries attached to the loading frame) show that there is not enough evidence to support a claim that a sample with seven cells has a higher $\square_{\max }$ than a sample of another number of cells. It was seen that $\square_{\max }$ can vary, sometimes greatly, from one test to another for similar samples. Figure 4 shows the average $\square_{\max }$ and $\square_{\text {plateau }}$ for all the samples tested $(\mathrm{N}=1-14)$. Observing the results in Figure 4, we notice that the $\square_{\text {plateau }}$ was much more consistent than $\square_{\max }$. From this we deduced that $\square_{\max }$ is highly sensitive to imperfections in the samples. Since the maximum load corresponds to a highly unstable buckling type event, it makes sense that $\square_{\max }$ (directly correlated to the maximum buckling load) would be imperfection sensitive and different in every test.

\section{B. Stability Analyses}

After it was determined that imperfections in the honeycomb samples had a large effect on the results, samples were studied to see if there was a correlation between a sample's shape and the imperfection sensitivity of that sample. Table 1 contains sketches of sample shapes, and number of lines of symmetry. Symmetry is based on the geometric symmetry of the figures shown. By comparing Table 1 to the scaling results from Figure 4 it can be seen that the more lines of symmetry a sample contains, the more likely it is to have a smaller error range. A more symmetric sample is less sensitive to imperfections, which causes the sample to perform similarly every time it is loaded.

Another way to characterize the spatial distribution of material in a cellular structure like honeycomb is to use the notion of the fabric tensor ${ }^{4}$. However, for such a characterization, one needs to have a sufficiently large number of cells within the cross-section to construct the fabric tensor. For samples with $\mathrm{N}=7$ or greater and assuming radial periodicity every $60^{\circ}$, the fabric tensor for the circular cell honeycombs that have been studied is shown in Figure 5 .

\section{Energy Absorption}

Since honeycomb structures are often used in an energy absorption role, the scaling of energy absorption with honeycomb size was studied. Using the loading-unloading data obtained during experiments, the energy absorbed per unit of permanent deformation was calculated. Energy absorbed was calculated as the area under the loaddisplacement curve. The permanent deformation was considered to be the length that a honeycomb had shortened once the load had been reduced to zero after crushing. The results are presented in Figure 6.

Figure 6 shows that there does not appear to be a scaling effect of energy absorption. This is because it depends mostly on plateau stress, and this stress is found to be independent (within statistical significance) of the number of cells, N. Since the plateau stress is independent of cell size, different honeycomb sizes and configurations do not have a great effect on this quantity.

\section{Finite Element Analysis}

In order to check the accuracy of the laboratory tests, a finite element model of the honeycomb was developed using the commercial software packages SDRC-IDEAS and Abaqus. Thin shell, eight-noded elements were used to create a seven-cell honeycomb model. This model was used to determine the proper material and simulation properties that would be used in future FEA simulations. After it was determined that these model and simulation properties were satisfactory, models containing $1,3,4,5$, and 9 cells were created and analyzed.

The analyses were carried out in Abaqus using nonlinear geometry and the Riks method option. The Riks method recalculates the model stiffness matrix at every specified time step allowing all equilibrium solutions (stable and unstable) to be captured. The models were analyzed using boundary displacement loading as was done in the experiments. A picture of the original seven-cell model before and after loading is shown in Figure 7. The picture shows that deformation predicted in the FEA simulations is similar to what was seen in the experiments.

As stated earlier, with buckling problems, initial geometric imperfections have a strong effect on the maximum buckling load. It is also known that non-linearity in the material response curve also has an effect on the maximum buckling load. These two properties were studied and altered accordingly until the resulting FEA response closely matched the experimental response.

Geometric imperfection was introduced to the model by performing an eigenmode analyses in Abaqus to determine the lowest 3 axial buckling modes. The mode corresponding to the smallest eigenvalue was used as an 
initial imperfection shape and the "perfect" shell mesh was perturbed by a pre-determined magnitude of this lowest eigenmode shape. It was determined that an imperfection magnitude of $0.2 \%$ of the shell thickness produced the best agreement with the experimental results.

Material non-linearity was introduced to the model using $\mathrm{J} 2$ incremental theory of plasticity ${ }^{3}$. To determine the material properties of polycarbonate, several tensile loading tests were performed on single circular polycarbonate cells. The results are shown in Figure 8 along with polycarbonate properties that were presented in Ref. 2. From these results, an acceptable range of material stiffness was determined. Using J2 incremental theory of plasticity the material response was modeled using a piecewise linear uniaxial stress-strain curve which is also shown in Figure 8. Initially, the slopes of the elastic and plastic parts of the curve were held as variables. Many test simulations were done to determine the slopes that produced the closest agreement with experiment. Ultimately it was found that slopes of $273 \mathrm{Ksi}$ for the elastic part of the curve and $53 \mathrm{Ksi}$ for the plastic part of the curve generated the best similarity to the experimental results.

A graph comparing the FEA results to three sets of experimental results is shown in Figure 9. The FE results predict a maximum load of approximately $75 \mathrm{lbs}$., which is in the range of the experimentally measured values. The simulation was unable to properly predict the plateau load that was seen during experiments. An investigation into reasons for this discrepancy is ongoing. After the finite element model and the method used to analyze it were verified for the seven cell honeycomb, the model was expanded for other sizes of honeycomb. Figure 10 shows the scaling results for $\square_{\max }$ for both experiments and finite element simulations. The graph shows good correlation between the results.

\section{Conclusion}

The effect of out of plane crushing on circular cell polycarbonate honeycombs has been studied experimentally and numerically. The results show that number of cells does not have an effect on the compressive strength (per unit area) of the honeycomb, but that the way that the cells are arranged does have an effect on the response. Samples that were determined to be "more stable" are less sensitive to imperfection and produce results that are less varied. Finite element analysis was used to simulate the behavior of the honeycombs during crushing. The FEA results are in good agreement with the experimental results for the six different honeycomb sizes that were studied.

\section{References}

${ }^{1}$ E. C. Mellquist and A. M. Waas, "Size Effects In the Compressive Crushing Of Honeycombs", AIAA SDM 2002

${ }^{2}$ J. Chung and A. M. Waas, "In-plane biaxial crush response of polycarbonate honeycombs", J. Engrg. Mech., ASCE, Vol. 127, No. 2, pp. 180, 2001

${ }^{3}$ J. Chung and A. M. Waas, "Compressive response and failure of circular cell polycarbonate honeycombs under inplane uniaxial stresses", J. Eng. Mat. Tech., Vol. 121, No. 4, pp. 494, 1999.

${ }^{4}$ M. Oda, J. Konishi and S. Nemat-Nasser, "Some experimentally based fundamental results on the mechanical behavior of granular materials",Geotechnique., Vol. 30, pp. 479, 1980. 


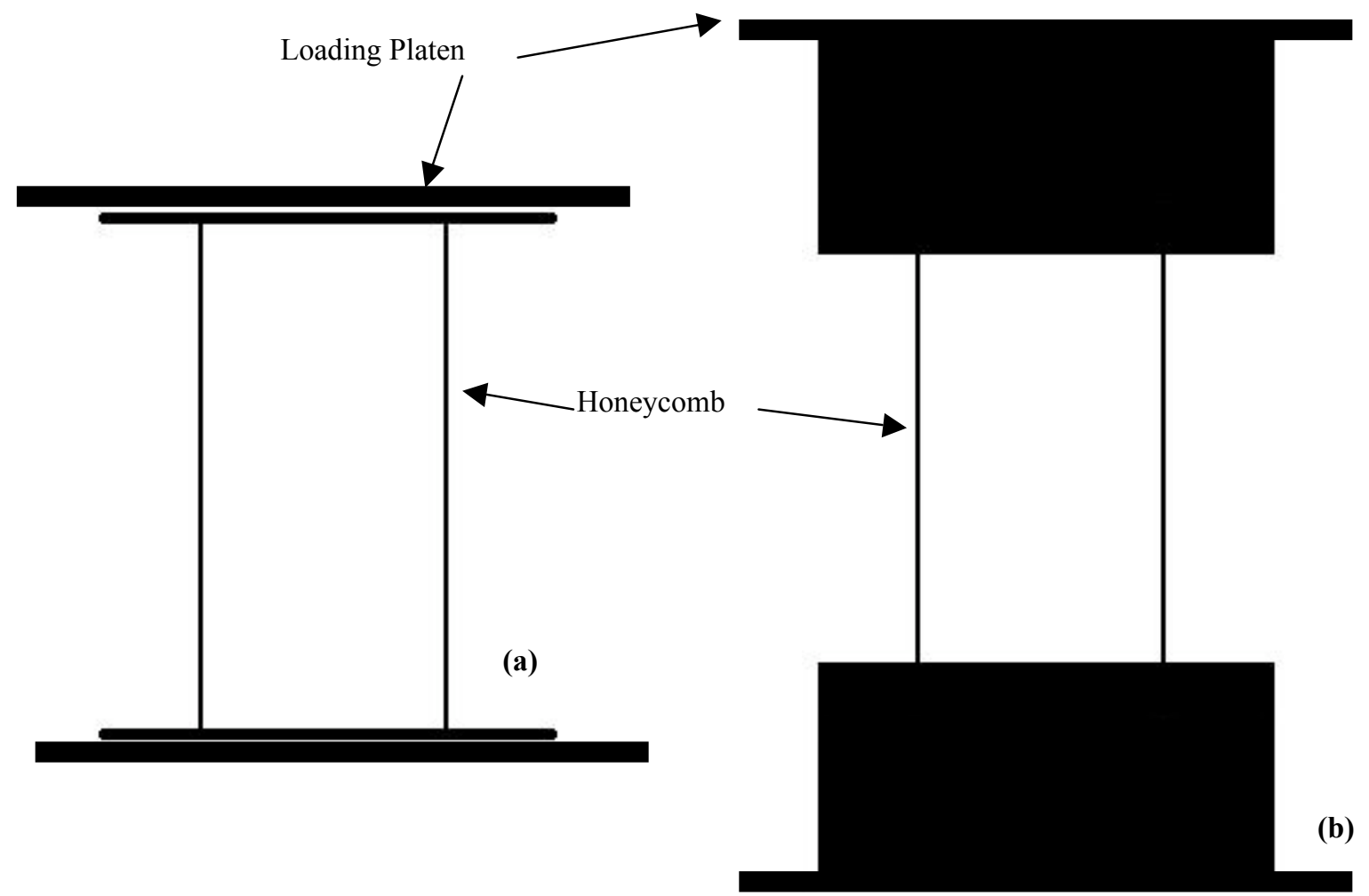

Figure 1. Original and New Test Setup. In the original setup (a), the honeycomb was attached to flat plates, which were not attached to the loading platen. In the new setup (b), the honeycomb was set in ( $1 / 8^{\text {th }}$ in deep) loading blocks, which were rigidly attached to the loading platen.
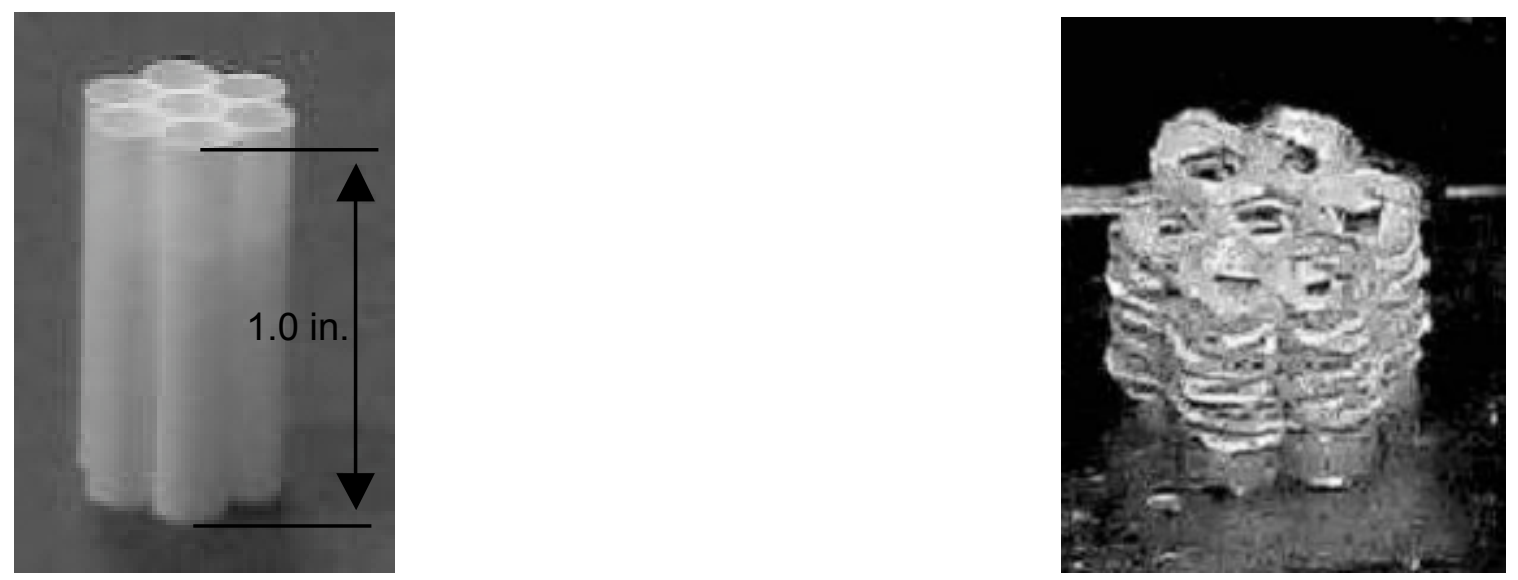

Figure 2. Undeformed and Deformed Honeycombs $(\mathrm{N}=7)$. 


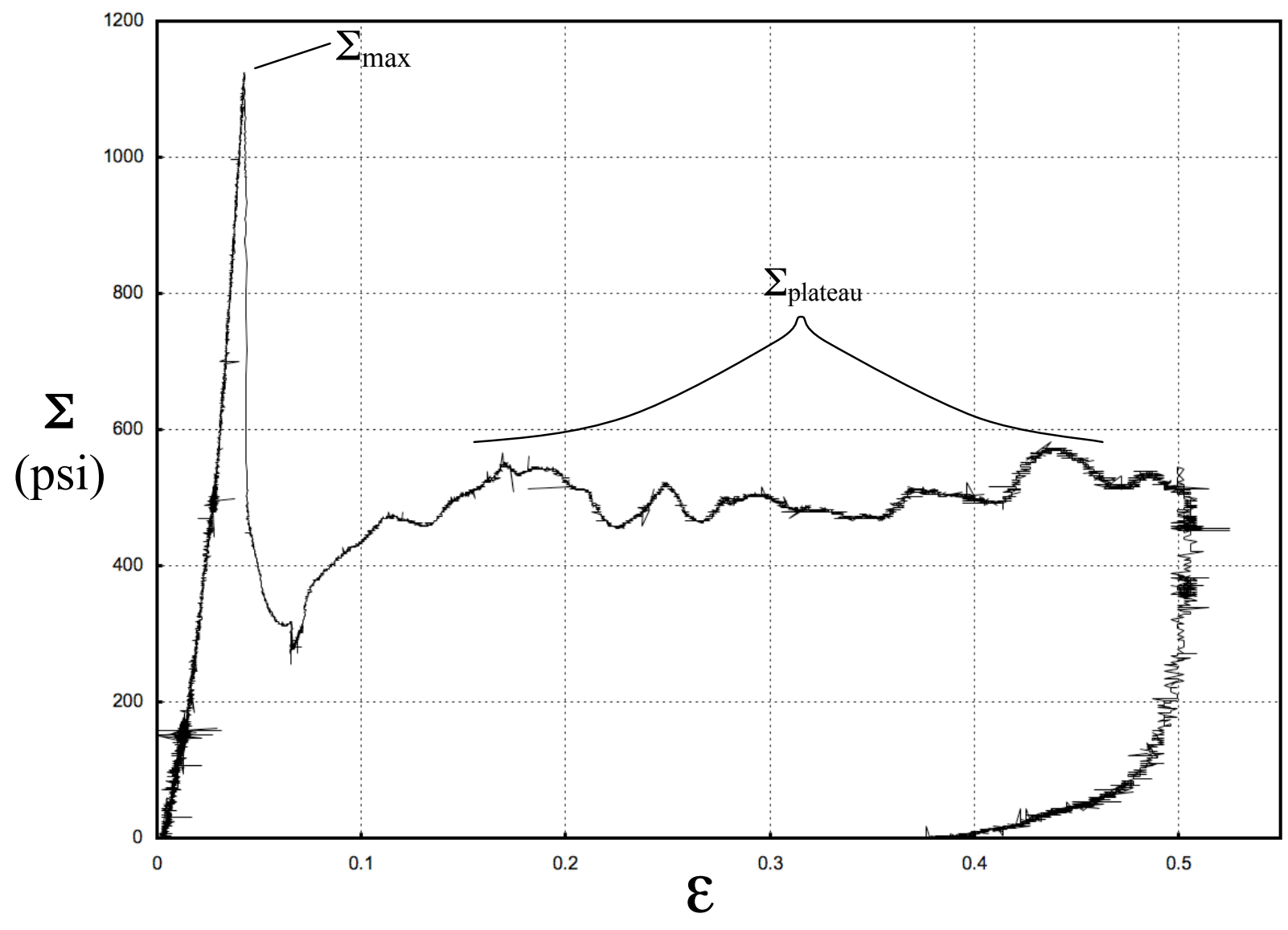

Figure 3. A typical response diagram for $\mathrm{N}=7$. 


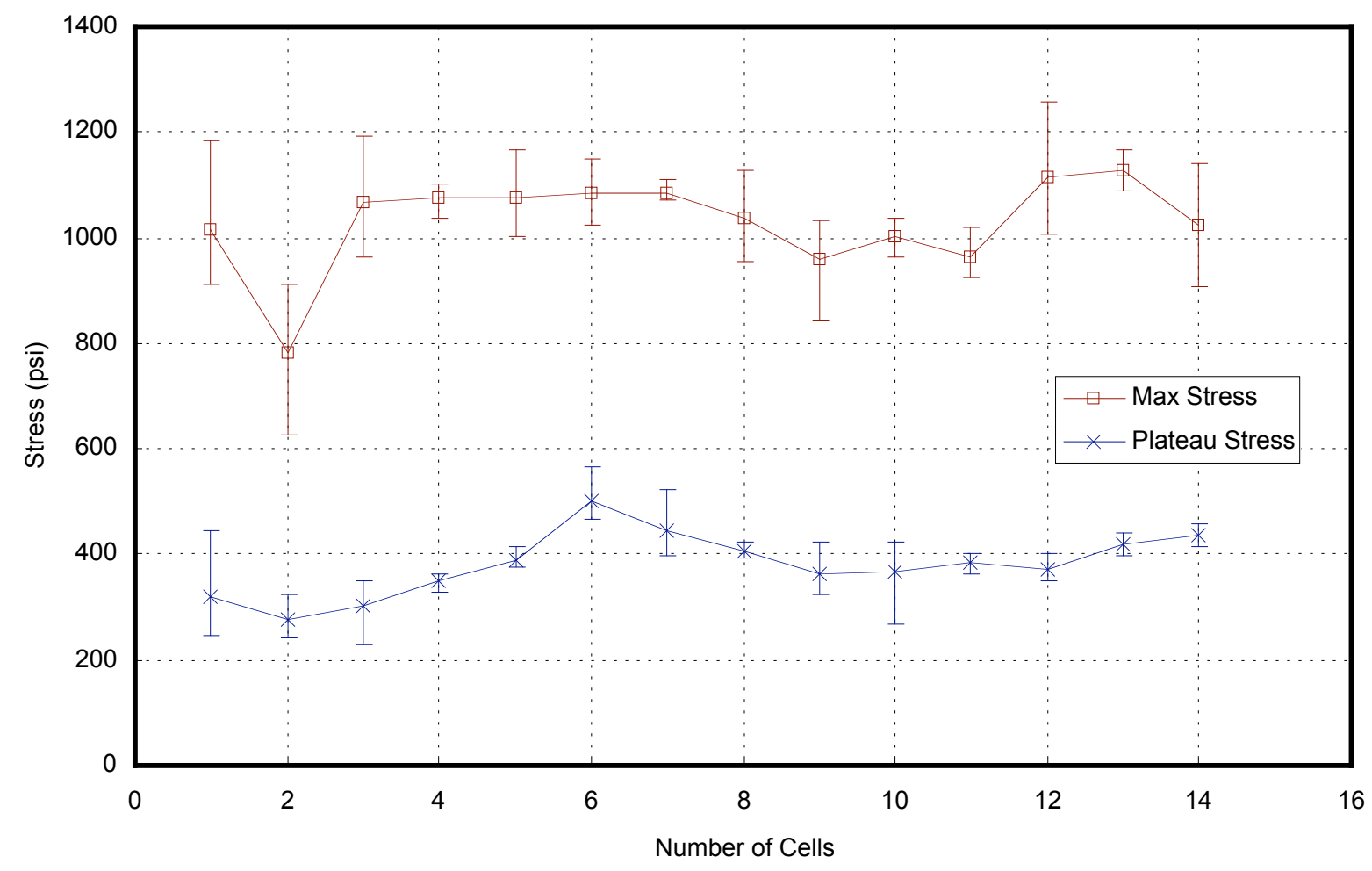

Figure 4. Scaling Of Honeycomb Properties. Both $\square_{\max }$ and $\square_{\text {plateau }}$ are shown. The error bars indicate the range of results for a given number of cells. The error for the plateau stress is much lower than for maximum stress indicating that $\square_{\max }$ is more sensitive to imperfections in the sample. 


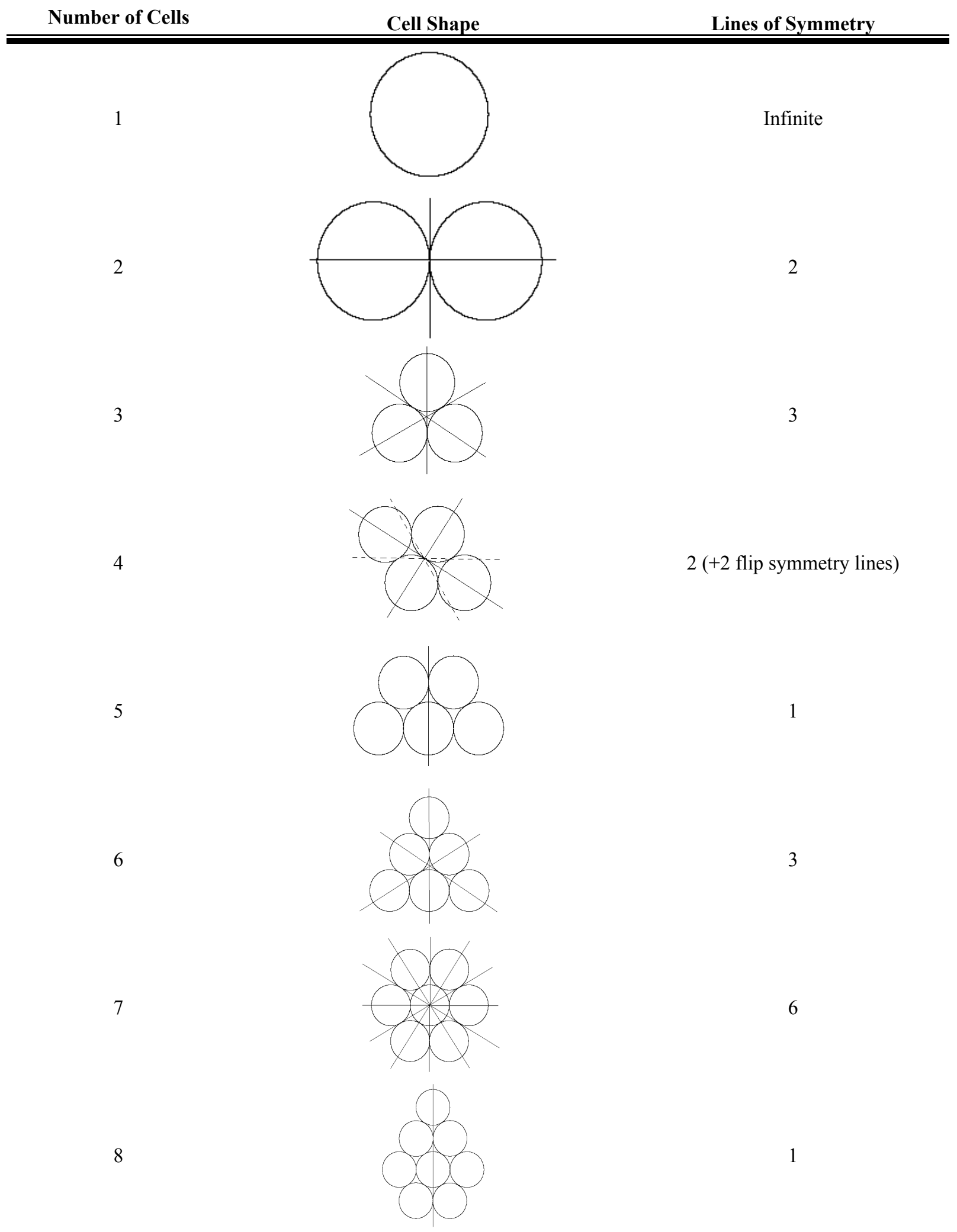


9
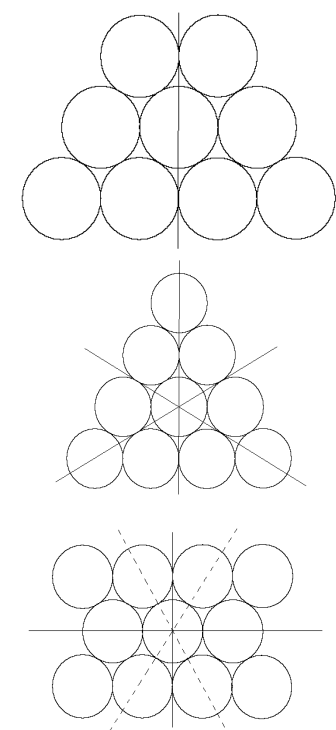

2 (+2 flip symmetric lines)

12

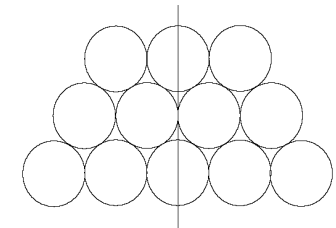

1

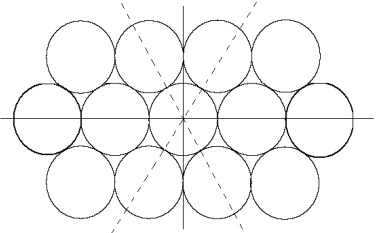

2 (+2 flip symmetric lines)

13

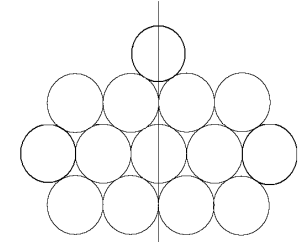

1 symmetry. Dashed lines indicate lines of "flip symmetry". These lines go through the geometric centroid of the sample and leave equal amounts of area on both sides of the line. 


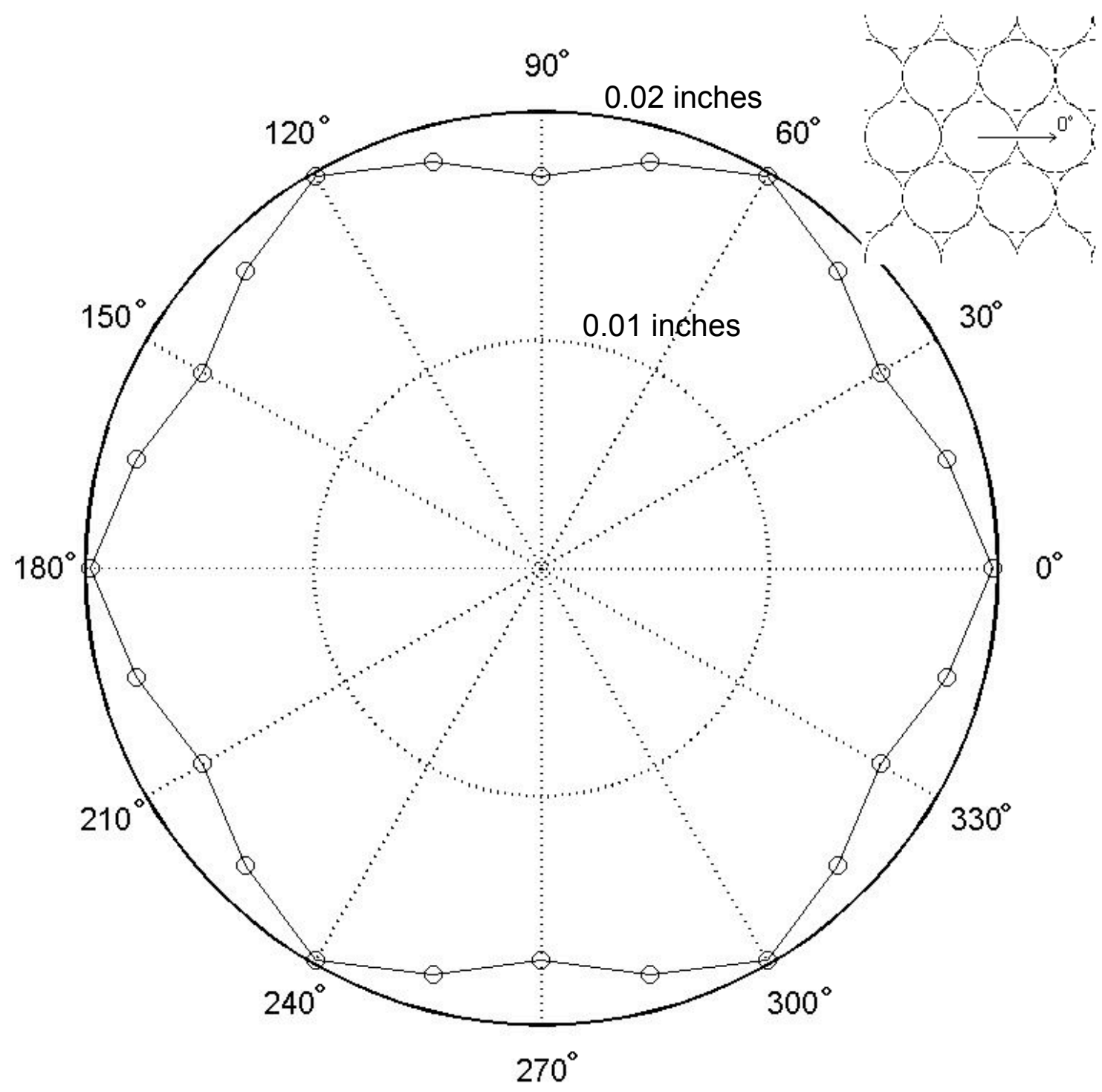

Figure 5. The Fabric Tensor For a Honeycomb Sample With an Infinite Number Of Cells. The mean intercept length is shown based on direction. The inset picture shows the direction indicated as $0^{\circ}$. 


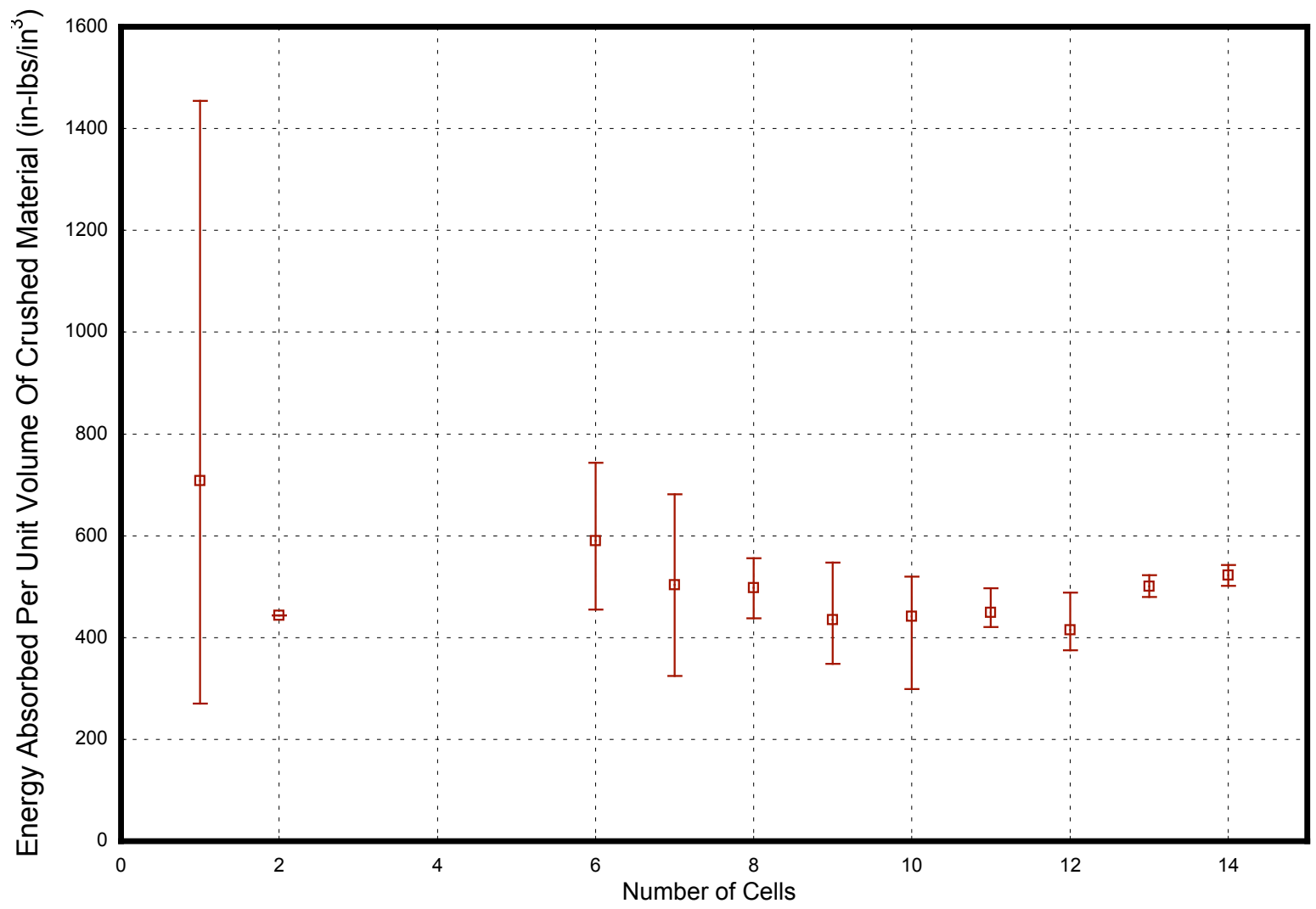

Figure 6. Scaling of Energy Absorption. The graph shows that the energy that a honeycomb absorbs during crushing is not affected by the number of cells. 

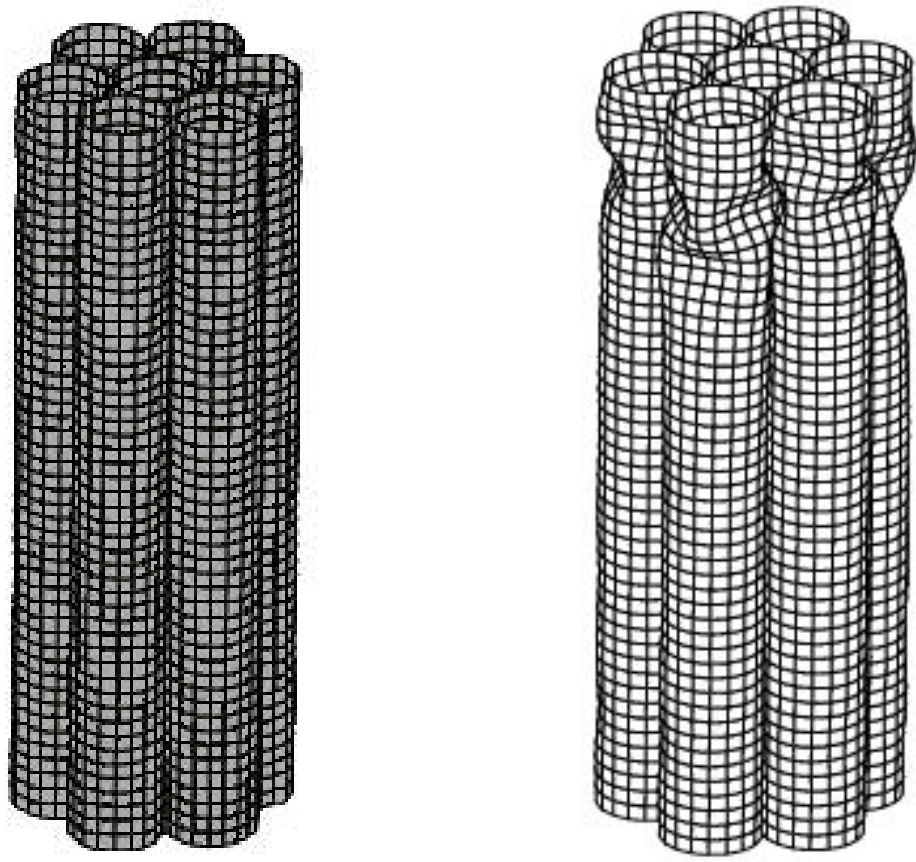

Figure 7. Undeformed And Deformed Finite Element Models. The slight eigenmode imperfection can be seen in the undeformed model. The deformed model shows the same buckle pattern that was seen in the experiments.

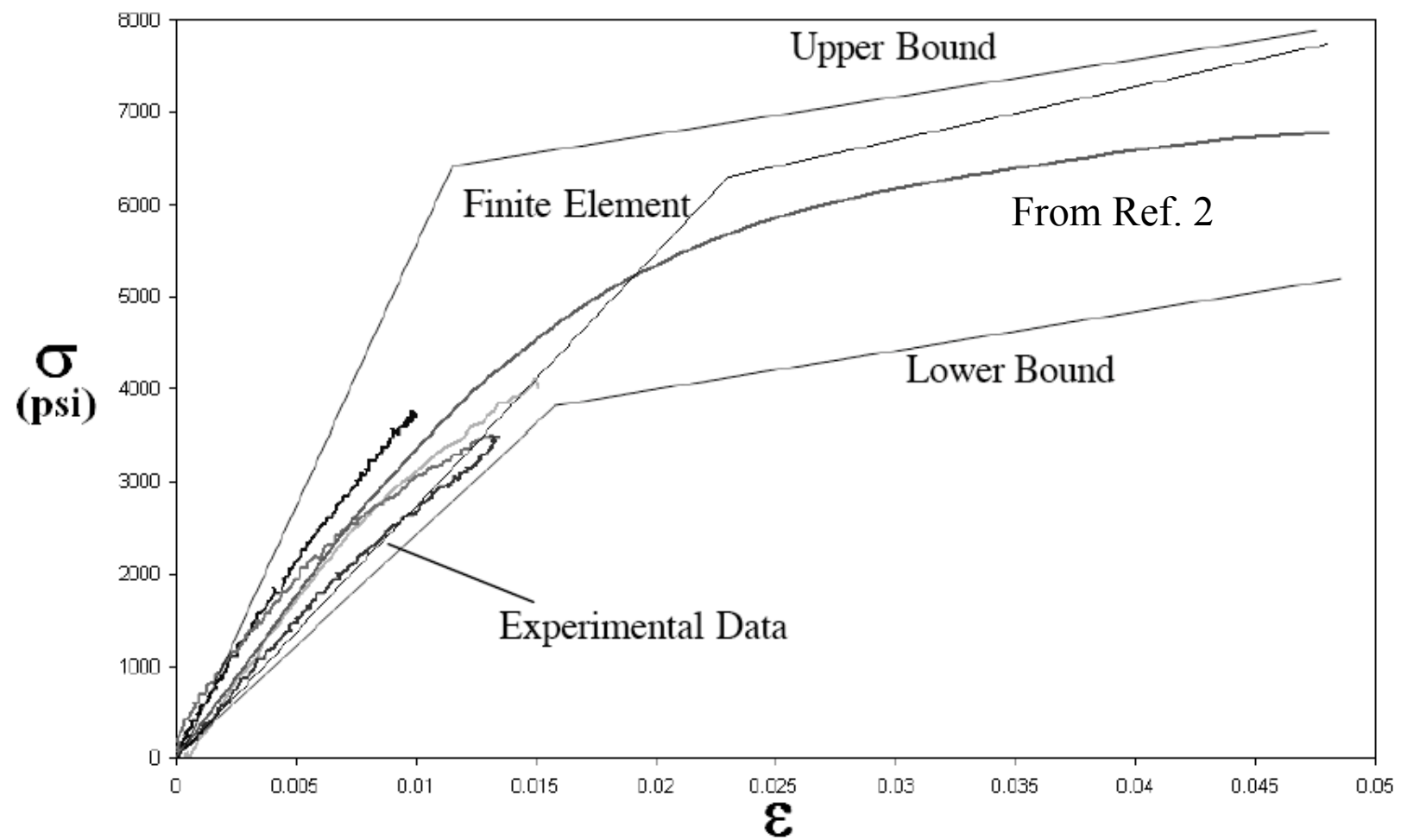

Figure 8. Material Properties Of Polycarbonate. Based on experimental data and the curve from Ref. 2 an upper and lower bound for material properties were established. By working within these boundaries, a suitable material properties curve was found for the finite element analysis. 


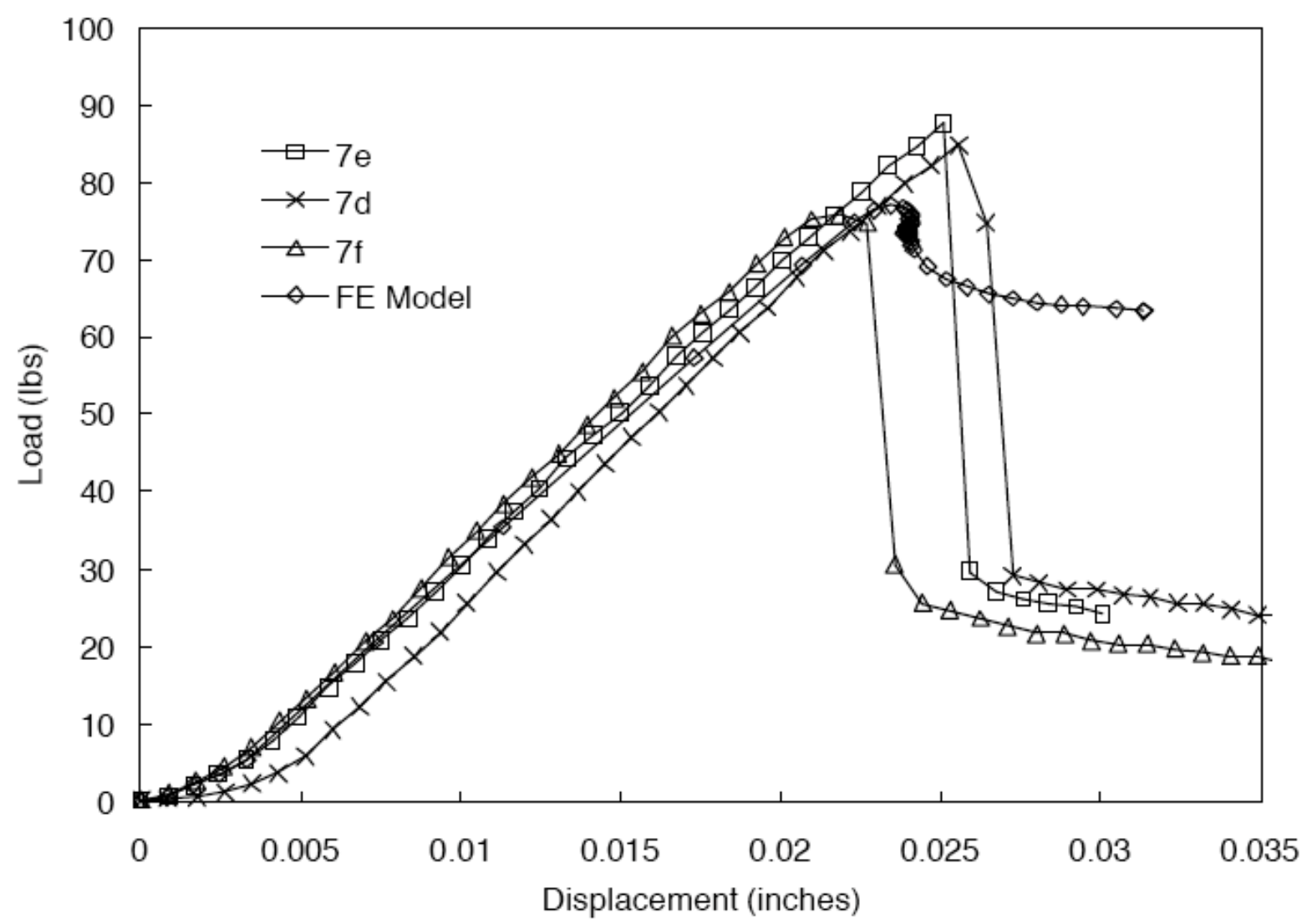

Figure 9. Comparison Of Finite Element And Experimental Results. The FEA results show good prediction of the maximum load, but are unable to capture the plateau load results. 


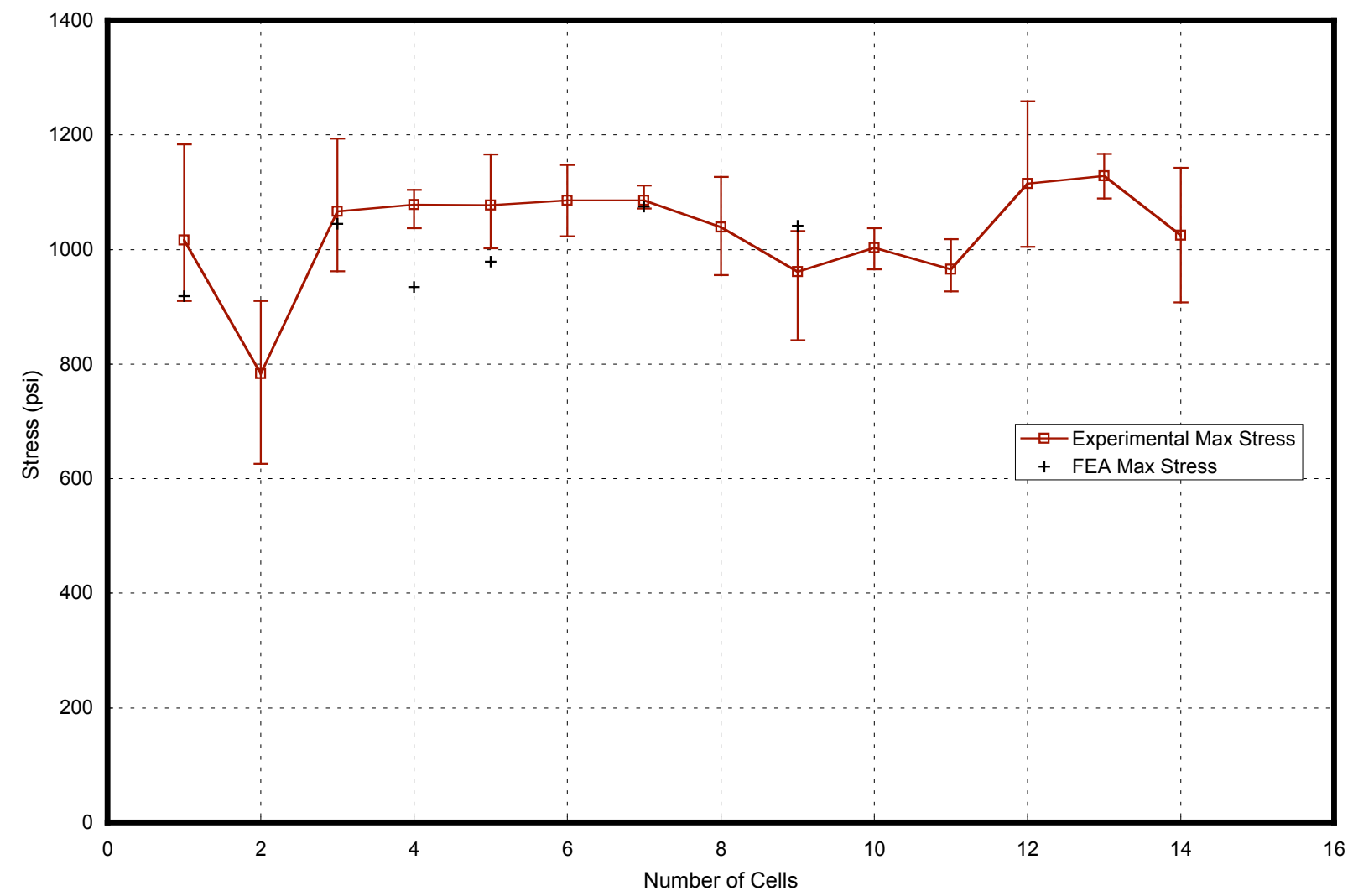

Figure 10. Maximum Load Scaling For Finite Element Results. The FEA results correlate well with the experimental results. 